\title{
ON THE ALGEBRA OF NETWORKS
}

\author{
BY
}

R. BOTT(1) AND R. J. DUFFIN

1. Introduction. This paper is a study of the constrained inverse of an arbitrary matrix $G$. The notion of a constrained inverse was suggested by electrical network theory. Conversely the results of this paper give an abstract foundation for the algebraic part of network theory.

In the analysis of many electrical and mechanical systems a matrix $G$ appears as a "metric" in a quadratic form

I.

$$
E=\sum_{1}^{n} \sum_{1}^{n} G_{i j} v_{i} v_{j}
$$

The matrix elements $G_{i j}$ and the coordinates $v_{i}$ have a direct physical interpretation. $E$ is related to energy or power. The state of the system is determined by a variational principle involving $E$. If the variations $\delta v_{i}$ are arbitrary, then it is clear that $G$ and its inverse would play a fundamental role. However, it often happens that the coordinates are subject to $n-m$ homogeneous linear constraints. The usual procedure, in this case, is to express the $v_{i}$ in terms of a set of unconstrained coordinates $q_{i}$. Hence

II.

$$
E=\sum_{1}^{m} \sum_{1}^{m} A_{i j} q_{i} q_{j}
$$

In this formulation the matrix $A$ would replace $G$ in importance. For a single problem in which the $G_{i j}$ have fixed numerical values there is an advantage to this method in that fewer variables are involved. It is the method almost always employed in numerical analysis. There is a great deal of arbitrariness in the choice of the coordinates $q_{i}$. Thus the matrix elements $A_{i j}$ are not invariants. Moreover they do not have such a direct physical interpretation as do the $G_{i j}$. The concern here is with algebraic relations and not with numerical analysis. It is clear, therefore, that the basic relations should involve the $G_{i j}$ and not the $A_{i j}$.

To restore $G$ to its position of importance we return to the formulation I. Let $V$ be the subspace to which the coordinates are constrained. The discriminant $D$ is defined as the determinant of $G$ relative to $V$. It is a homogeneous multilinear function of the $G_{i j}$. The constrained inverse $T$ of $G$ is an

Presented to the Society, December 30, 1948; received by the editors March 31, 1952.

(1) The material in this paper was submitted as part of a thesis by the first author to the Carnegie Institute of Technology, June 1, 1949 in partial fulfillment of the requirements for the degree of Doctor of Science. 
$n$ by $n$ matrix such that $T$ is a transformation into $V$ and $T G$ is the identity in $V$. The constrained inverse has many properties analogous to those of the ordinary inverse. For example $G$ has a constrained inverse if and only if $D \neq 0$. The variational problem is completely solved by $T$.

If $G^{\prime}$ is another matrix, complementary expressions are defined relative to the subspace $V^{\prime}$ which is the orthogonal complement of $V$. Interesting relationships exist if $G^{\prime}=G^{-1}$. This concept of complementarity throws new light on the physical applications.

Of particular significance is the case that $G$ is a diagonal matrix. Let the diagonal elements be $g_{1}, g_{2}, \cdots, g_{n}$. Let $\psi=\log D, \psi_{i}=\partial \psi / \partial g_{i}$, and $\psi_{i j}$ $=\partial^{2} \psi / \partial g_{i} \partial g_{j}$. Then $-\psi_{i j}$ is the square of a rational function. This generalizes a result of Richard Cohn. Conversely any homogeneous multilinear function which satisfies this perfect square condition is, within a constant factor, the discriminant of a diagonal matrix. From the viewpoint of pure mathematics this is the most interesting result of this paper. All of the derivatives of $\psi$ are expressible in terms of the matrix elements of $T$. Thus $\psi_{i}=T_{i i}$ and $\psi_{i j}=-\left(T_{i j}\right)^{2}$.

The theory developed here has a close relationship with the work of G. A. Campbell, G. Kron, and Richard Cohn. Campbell [2] (2) is concerned with the use of the discriminant and the variational method. Kron $[7 ; 8]$ is concerned with the notion of invariance and general matrix methods. Through correspondence with H. W. Becker we learned of Cohn's observation that the derivative of the joint resistance of a network with respect to the resistance of any branch is a perfect square. A converse to this theorem is described above. Independently Cohn [3] also obtained a converse. Our results and his do not overlap; taken together they give an interesting characterization of network functions.

2. The constrained inverse of a matrix. Let $U$ be a real vector space of $n$ dimensions with a fixed coordinate system. Then a sequence of real numbers $u_{1}, u_{2}, \cdots, u_{n}$ may be regarded as a vector $u$ of $U$. Let $(u, v)$ designate $\sum_{1}^{n} u_{i} v_{i}$. If $(u, v)=0$, then the vectors $u$ and $v$ are said to be orthogonal.

Let $V$ and $V^{\prime}$ be orthogonal complementary subspaces of $U$. If $V$ has dimension $m$ and $V^{\prime}$ has dimension $m^{\prime}$ then $m+m^{\prime}=n$. An arbitrary vector $u$ has a unique decomposition in the form

$$
u=v+v^{\prime} ; \quad v \in V, \quad v^{\prime} \in V^{\prime} .
$$

Let $P u=v$ and $P^{\prime} u=v^{\prime}$; then $P$ and $P^{\prime}$ are the well known perpendicular projection transformations of $U$ into $V$ and $V^{\prime}$ respectively. It follows from (1) that

$$
P=P^{2}=\tilde{P}, \quad P P^{\prime}=P^{\prime} P=0, \quad P+P^{\prime}=I .
$$

Thus $P$ may be regarded as a real symmetric matrix whose square is itself.

(2) Numbers in brackets denote references cited at the end of the paper. 
A sequence of complex numbers $u_{1}, \cdots, u_{n}$ may be termed a complex vector. We say that a complex vector is in $V$ if its real and imaginary parts are in $V$. In terms of this convention it is clear that (1) is still valid.

DEFINITIONS:

(a) $G$, an arbitrary complex $n$ by $n$ matrix.

(b) $D=\operatorname{det}\left(G P+P^{\prime}\right)$, the discriminant of $G$ in $V$.

(c) $T=P\left(G P+P^{\prime}\right)^{-1}$, the constrained inverse of $G$.

(d) $\psi=\log D$, the potential of $T$.

If $G$ has an inverse, let it be denoted by $G^{\prime}$. Then complementary expressions are defined as: $D^{\prime}=\operatorname{det}\left(G^{\prime} P^{\prime}+P\right), T^{\prime}=P^{\prime}\left(G^{\prime} P^{\prime}+P\right)^{-1}, \psi^{\prime}=\log D^{\prime}$.

THEOREM 1. Let $h$ be an arbitrary vector. Then if $D \neq 0$, the relation

$$
G v+v^{\prime}=h ;
$$$$
v \in V, v^{\prime} \in V^{\prime},
$$

has the unique solution

$$
\begin{aligned}
v & =T h, \\
v^{\prime} & =(I-G T) h .
\end{aligned}
$$

If $D=0$, there is an $h$ such that (3) has no solution.

Proof. Define $X=\left(G P+P^{\prime}\right)$ and $Y=X^{-1}$. Write $u=v+v^{\prime}$; then (3) may be written in the form $X u=h$. Thus $u=Y h$ and $v=P Y h=T h$ and $v^{\prime}=P^{\prime} Y h$ $=(X-G P) Y h=(I-G T) h$. The last statement of the theorem is now obvious because, if $D=0$, then $X$ is singular.

The fundamental equation of network theory is

$$
G v+v^{\prime}=G e+e^{\prime}
$$

where $e$ and $e^{\prime}$ are arbitrary vectors. By Theorem 1 the solution is

$$
\begin{gathered}
v=T G e+T e^{\prime}, \\
v^{\prime}=(I-G T) e^{\prime}+(G-G T G) e .
\end{gathered}
$$

Theorem 2. If $D \neq 0, P=T G P=P G T$ and $T=P T=T P$.

Proof. From (c), $P T=T$. Multiply $T\left(G P+P^{\prime}\right)=P$ on the right by $P$ so $T G P=P$. Hence $T P^{\prime}=0$ and $T P=T$. Multiply (5) by $P$ so $(P-P G T) h=0$ or $P=P G T$.

Theorem 3. If $D \neq 0, \partial \psi / \partial G_{i j}=T_{j i}$ and $\partial T_{a b} / \partial G_{i j}=-T_{a i} T_{j b}$.

Proof. This theorem is well known if $T$ were the true inverse of $G$. Let a dagger denote partial differentiation with respect to the matrix element $G_{i j}$. Then $T X=P$ so $T \dagger X+T X \dagger=0$ or $T \dagger=-T X \dagger Y$. But $X \dagger=G \dagger P$ so

$$
T \dagger=-T G \dagger T \text {. }
$$

Again $\psi \dagger=\sum_{r} \sum_{s}\left(\partial \psi / \partial X_{r s}\right) X_{r s}^{\dagger}=\sum_{r} \sum_{s} Y_{s r} X_{r s}^{\dagger}=$ trace $X \dagger Y$. Substituting 
$X \dagger=G \dagger P$ gives

$$
\psi \dagger=\operatorname{trace} G \dagger T .
$$

Clearly (9) and (10) are equivalent to Theorem 3.

Theorem 4. If $D \neq 0$ and $\operatorname{det} G \neq 0$, then $D^{\prime}=D / \operatorname{det} G$ and $T^{\prime}=G-G T G$.

Proof. $D=\operatorname{det}\left(G P+P^{\prime}\right)=\operatorname{det} G\left(P+G^{\prime} P^{\prime}\right)=(\operatorname{det} G) D^{\prime}$. Multiply equation (6) on the left by $G^{\prime}$ so

$$
G^{\prime} v^{\prime}+v=G^{\prime} e^{\prime}+e .
$$

Hence by the complement of (7), $v^{\prime}=T^{\prime} G^{\prime} e^{\prime}+T^{\prime} e$. Comparing this with (8) completes the proof because $e$ is arbitrary.

THEOREM 5. If $D \neq 0$ and $G$ is symmetric, then:

(a) $T$ is symmetric.

(b) $\partial^{2} \psi / \partial G_{i i} \partial G_{j j}=-\left(T_{i j}\right)^{2}$.

(c) The quadratic form $Q=(1 / 2)(G(v-e), v-e)-\left(e^{\prime}, v\right), v \in V$, has a unique stationary value when $v=T G e+T e^{\prime}$.

Proof. (a) By Theorem 2, $\left(P G+P^{\prime}\right) T=P$; hence $\tilde{T}\left(\widetilde{G} P+P^{\prime}\right)=P$, so if $\tilde{G}=G$, then $\tilde{T}=P\left(G P+P^{\prime}\right)^{-1}=T$. (b) By Theorem $3, \partial \psi / \partial G_{i i}=T_{i i}$ and $\partial T_{i i} / \partial G_{j j}=-T_{i j} T_{j i}$. (c) Clearly $\delta Q=\left(G(v-e)-e^{\prime}, \delta v\right)$ where the variation $\delta v$ may be an arbitrary vector of $V$. Choose $\delta v$ real. Hence if $\delta Q=0$, it follows that $G(v-e)-e^{\prime}$ is a vector of $V^{\prime}$. But this is precisely equation (6).

Theorem 6. If R.P. $(G u, \bar{u})>0$ for arbitrary $u \neq 0$, then $D \neq 0$ and R.P. $(T u, \bar{u}) \geqq 0$ and R.P. $T_{i i} \geqq 0$.

Proof. Here R.P. means "real part" and $\bar{u}$ means the complex conjugate of $u$. Suppose that $G v+v^{\prime}=0$. Then $(G v, \bar{v})=0$; hence $v=0$ and $v^{\prime}=0$. Thus $G P+P^{\prime}$ is not singular so $D \neq 0$. Let $u=v+v^{\prime}$ and let $s=(T u, \bar{u})=(T v, \bar{v})$. If $T v=v_{1}$, then $P G v_{1}=v$, so $s=\left(v_{1}, P \bar{G} \bar{v}_{1}\right)=\left(\bar{G} \bar{v}_{1}, v_{1}\right)$. Hence R.P. $\bar{s} \geqq 0$. The last part of the theorem is obtained by taking $u$ to have only one component.

Let $\left(C+C^{\prime}\right)$ be an $n$ by $n$ matrix whose first $m$ columns $C$ are real independent vectors of $V$ and whose last $n-m$ columns $C^{\prime}$ are real independent vectors of $V^{\prime}$. Likewise let $\left(K+K^{\prime}\right)$ be an $n$ by $n$ matrix whose first $m$ rows $K$ are real independent vectors of $V$ and whose last $n-m$ rows $K^{\prime}$ are real independent vectors of $V^{\prime}$. By independence

$$
\operatorname{det}\left(C+C^{\prime}\right) \neq 0, \quad \operatorname{det}\left(K+K^{\prime}\right) \neq 0 .
$$

By orthogonality

$$
K C^{\prime}=0, \quad K^{\prime} C=0 .
$$

Let $q$ represent a vector whose last $n-m$ components vanish. Then any vector $v \in V$ may be written 


$$
v=C q .
$$

Thus $C$ might be termed "a rectangular projection into $V$. "

Let $A=K G C$ and $A_{0}=K C$ be regarded as $m$ by $m$ matrices.

Theorem 7. If $m>0$, then $D=\operatorname{det} A / \operatorname{det} A_{0}$ and if $D \neq 0$, then $T=C A^{-1} K$.

Proof. Let $Z=\left(K+K^{\prime}\right)\left(G P+P^{\prime}\right)\left(C+C^{\prime}\right)=\left(K+K^{\prime}\right)\left(G C+C^{\prime}\right)=K G C$ $+K^{\prime} C^{\prime}+K^{\prime} G C=A+A_{0}^{\prime}+K^{\prime} G C$. If $0<m<n, Z$ has the form

$$
Z=\left|\begin{array}{cc}
A & 0 \\
K^{\prime} G C & A_{0}^{\prime}
\end{array}\right| .
$$

Thus $\operatorname{det} Z=\operatorname{det} A \operatorname{det} A_{0}^{\prime}$. For $G=I$ this gives $\operatorname{det}\left(K+K^{\prime}\right) \operatorname{det}\left(C+C^{\prime}\right)$ $=\operatorname{det} A_{0} \operatorname{det} A_{0}^{\prime}$. By (12) the left side is not zero so $\operatorname{det} A_{0} \neq 0$. Hence $D$ $=\operatorname{det} Z / \operatorname{det}\left(K+K^{\prime}\right) \operatorname{det}\left(C+C^{\prime}\right)=\operatorname{det} A / \operatorname{det} A_{0}$.

Equation (3) may be written by use of (14) in the form $G C q+v^{\prime}=h$. Thus $K G C q=K h, q=A^{-1} K h$ and $v=C A^{-1} K h$. Hence by Theorem 1 it follows that $T=C A^{-1} K$.

THEOREM 8. $D$ is a homogeneous multilinear form of degree $m$ as a function of the matrix elements $G_{i j}$ of $G$. The coefficients of terms containing only diagonal elements $G_{i i}$ are positive.

Proof. It may be supposed that $0<m<n$. The matrix elements of $A$ are $A_{r s}=\sum_{i} \sum_{j} K_{r i} G_{i j} C_{j s}$ and are homogeneous and linear in the $G_{i j}$. Thus det $A$ is homogeneous and of degree $m$ in the $G_{i j}$.

Let the various $m$ by $m$ minors of the rectangular matrix $K$ be denoted by $K^{a}, a=1,2, \cdots, p$, where $p=n ! / m !(m-n) !$. The minors of $C$ are ordered in a corresponding way. Let $G^{a b}$ be the $m$ by $m$ minor of $G$ which "meshes" with $K^{a}$ on the left and $C^{b}$ on the right.

Consider some particular nonvanishing term of $\operatorname{det} A$. The $m$ factors $G_{i j}$ of this term lie in no more than $m$ rows and in no more than $m$ columns of $G$. Thus all the factors appear in some minor, say $G^{a b}$. Let all the other matrix elements of $G$ vanish. In this case $A=K^{a} G^{a b} C^{b}$ and $\operatorname{det} A=\operatorname{det} G^{a b} \operatorname{det} K^{a}$ $\operatorname{det} C^{b}$. Thus $\operatorname{det} G^{a b} \neq 0$ so the $m$ factors all lie in different rows and in different columns of $G^{a b}$. It follows that these factors uniquely determine the minor $G^{a b}$. Thus the terms can be grouped according to minors of $G$. In other words

$$
\operatorname{det} A=\sum_{a} \sum_{b} \operatorname{det} G^{a b} \operatorname{det} K^{a} \operatorname{det} C^{b} .
$$

Clearly $\operatorname{det} A$ is multilinear in the $G_{i j}$.

The terms containing only diagonal elements of $G$ must arise from the diagonal of a minor of the form $G^{a a}$. Choose $K=\tilde{C}$ so the coefficient of this term is $\left(\operatorname{det} C^{a}\right)^{2} \geqq 0$. In this case $\operatorname{det} A_{0}=\sum_{a}\left(\operatorname{det} C^{a}\right)^{2} \geqq 0$. Making use of Theorem 7 completes the proof. 
3. The discriminant of a diagonal matrix. If $G$ is a diagonal matrix, let the diagonal element $G_{i i}$ be denoted by $g_{i}$. Then according to Theorem 8 the discriminant $D\left(g_{1}, \cdots, g_{n}\right)$ is a homogeneous multilinear form with positive coefficients. The necessity part of the following theorem is given by Theorem $5 \mathrm{~b}$ because $D(1,1, \cdots, 1)=1$.

THEOREM 9. Let $f$ be a homogeneous multilinear form as a function of the variables $g_{1}, g_{2}, \cdots, g_{n}$ which does not vanish identically and is such that

$$
\frac{\partial^{2} \log f}{\partial g_{i} \partial g_{j}}=-\left(S_{i j}\right)^{2}, \quad i, j=1, \cdots, n,
$$

where $S_{i j}$ is a real rational function. Then this condition is both necessary and sufficient that $f\left(g_{1}, g_{2}, \cdots, g_{n}\right) / f(1,1, \cdots, 1)$ be a discriminant of the $n$ by $n$ diagonal matrix $G$.

Proof. Let $\Psi=\log f$ and let the derivatives of $\Psi, f$, and $S_{i j}$ with respect to $g_{k}$ be denoted by $\Psi_{k}, f_{k}$, and $S_{i j k}$. In the neighborhood of any point, $f$ does not vanish identically. Thus given an $\epsilon>0$ there is a point $g_{i}=a_{i}, i=1, \cdots, n$, where $f$ does not vanish and $0<a_{i}<\epsilon$. It may be supposed that $f$ is positive at this point; otherwise $f$ could be multiplied by a suitable constant without changing the nature of the problem. Clearly $\Psi_{i}=f_{i} / f$ is a homogeneous function of degree -1 . Hence by Euler's theorem

$$
\Psi_{i}=-\sum_{1}^{n} \Psi_{i j} g_{j}=\sum_{1}^{n}\left(S_{i j}\right)^{2} g_{j}
$$

Thus $f_{i} / f \geqq 0$ and it follows that if $b_{i} \geqq a_{i}$, then $f(b) \geqq f(a)>0$. Hence $f>0$ if all $g_{i}>0$ because $\epsilon$ is arbitrary. In what follows it is assumed that all $g_{i}>0$. Clearly

$$
\Psi_{i j}=\left(f f_{i j}-f_{i} f_{j}\right) / f^{2}=-\left(S_{i j}\right)^{2}
$$

Let $q=S_{i j} f$; then

$$
\begin{gathered}
q^{2}=f_{i} f_{j}-f f_{i j}, \\
2 q q_{k}=f_{i} f_{j k}+f_{j} f_{i k}-f_{k} f_{i j}-f f_{i j k} .
\end{gathered}
$$

The right side of (18) is a polynomial of the second degree at most as a function of $g_{k}$. Hence $q$, being rational, must be a linear function of $g_{k}$. Divide both sides of (18) by $g_{k}^{2}$ and let $g_{k} \rightarrow \infty$; this gives

$$
q_{k}^{2}=f_{i k} f_{j k}-f_{k} f_{i j k} \text {. }
$$

Since $S_{i j k}=\left(f q_{k}-f_{k} q\right) / f^{2}$, then

$$
\left(f^{2} S_{i j k}\right)^{2}=f^{2} q_{k}^{2}-2 f f_{k} q q_{k}+f_{k}^{2} q^{2} .
$$

Substituting (18), (19), and (20) in (21) gives 


$$
\begin{aligned}
\left(f^{2} S_{i j k}\right)^{2}= & \left(f^{2} f_{i k} f_{j k}-f^{2} f_{k} f_{i j k}\right)+\left(-f f_{k} f_{i} f_{j k}-f f_{k} f_{j} f_{i k}+f f_{k}^{2} f_{i j}+f^{2} f_{k} f_{i j k}\right) \\
& +\left(f_{i} f_{j} f_{k}^{2}-f f_{k}^{2} f_{i j}\right) \\
= & f^{2} f_{i k} f_{j k}-f f_{k} f_{j} f_{i k}-f f_{k} f_{i} f_{j k}+f_{i} f_{j} f_{k}^{2} \\
= & \left(f f_{i k}-f_{i} f_{k}\right)\left(f f_{j k}-f_{j} f_{k}\right) .
\end{aligned}
$$

This proves that

$$
\left(\partial S_{i j} / \partial g_{k}\right)^{2}=\left(S_{i k} S_{j k}\right)^{2} .
$$

We now make use of the following:

Lemma. A polynomial $f\left(g_{1}, g_{2}, \cdots, g_{r}\right)$ which is of the first degree in each variable is irreducible if and only if $\left(\partial^{2} / \partial g_{i} \partial g_{j}\right) \log f$ does not vanish identically for $i, j=1,2, \cdots, r$.

Proof. Suppose that $f$ is reducible; then $f=h k$ where $h$ is of the first degree in $g_{i}$ and $k$ is of the first degree in $g_{j}$ for some $i \neq j$. Thus $f_{i} f_{j}-f f_{i j}=\left(h_{i} k\right)\left(h k_{j}\right)$ $-(h k)\left(h_{i} k_{j}\right) \equiv 0$.

Conversely suppose $f_{i} f_{j}-f f_{i j} \equiv 0$. If $f_{i j} \equiv 0$, then $f_{i} f_{j} \equiv 0$. Thus $f_{i} \equiv 0$ or $f_{j} \equiv 0$ which is impossible. So $f=f_{i} f_{j} / f_{i j}$. By the unique factorization theorem $f_{i j}=p_{i} p_{j}$ where $f_{i} / p_{i}$ and $f_{j} / p_{j}$ are polynomials. But $p_{i}$ and $p_{j}$ are of degree 0 in $g_{i}$ and $g_{j}$; hence $f_{i} / p_{i}$ is of degree 1 in $g_{j}$ and $f_{j} / p_{j}$ is of degree 1 in $g_{i}$ and $f=\left(f_{i} / p_{i}\right)\left(f_{j} / p_{j}\right)$ is reducible. Note that $f_{i j} \equiv 0$ if $i=j$.

Returning to the proof of Theorem 9, suppose that $f$ is expressed as a product of irreducible factors. Then this separates the variables into disjoint classes corresponding to the various factors and possibly a remainder class consisting of the variables which do not appear in $f$. Consider the variables which appear in the first factor. Then from (22)

$$
S_{i j k}=-\delta_{i j k} S_{i k} S_{j k}
$$

where $\delta_{i j k}=+1$ or -1 . By virtue of the lemma, $S_{i k}$ and $S_{j k}$ do not vanish identically; hence $S_{i j k}$ does not vanish identically. Thus $\delta_{i j k}$ is actually defined by (23) and is not indeterminate. But $\Psi_{i j}=-\left(S_{i j}\right)^{2}$, so

$$
\Psi_{i j k}=2 \delta_{i j k} S_{i j} S_{i k} S_{j k} .
$$

It may be supposed that $S_{i j}=S_{j i}$; moreover, the left side of (24) is symmetric in $i, j$, and $k$ so $\delta_{i j k}=\delta_{j i k}=\delta_{j k i}$, etc. Note that it has not been assumed that $i, j$, and $k$ are necessarily distinct. Thus $\delta_{i i j}$ and $\delta_{i i i}$ are defined.

Differentiating (23) with respect to $g_{h}$ gives

$$
S_{i j k h}=S_{k h}\left(\delta_{h} \delta_{j} S_{i h} S_{j k}+\delta_{h} \delta_{i} S_{i k} S_{j h}\right) .
$$

Here $\delta_{h}=\delta_{i j k}, \delta_{i}=\delta_{j k h}$, etc. But $S_{i j k h}=S_{i j h k}$ so that

$$
0=S_{k h}\left[\left(\delta_{h} \delta_{j}-\delta_{k} \delta_{i}\right) S_{i h} S_{j k}+\left(\delta_{h} \delta_{i}-\delta_{k} \delta_{j}\right) S_{i b} S_{j h}\right] .
$$


In this expression permute $i$ with $h$ and $j$ with $k$ so that

$$
0=S_{j i}\left[\left(\delta_{k} \delta_{i}-\delta_{h} \delta_{j}\right) S_{i h} S_{j k}+\left(\delta_{h} \delta_{i}-\delta_{k} \delta_{j}\right) S_{j h} S_{i k}\right] .
$$

Multiply the first expression by $S_{j i}$ and the second by $S_{k h}$ and add. This gives $0=\left(\delta_{h} \delta_{i}-\delta_{k} \delta_{j}\right) S_{j i} S_{k h} S_{i k} S_{j h}$. By the lemma it follows that $\left(\delta_{h} \delta_{i}-\delta_{k} \delta_{j}\right)=0$ or

$$
\delta_{i j k}=\delta_{i j h} \delta_{j k h} \delta_{k i h} .
$$

Choose some variable of the first factor to have a special significance, say $g_{h}$. Then define $T_{i j}=\delta_{i j h} S_{i j}$. By virtue of (26) relation (23) may be written as

$$
\partial T_{i j} / \partial g_{k}=-T_{i k} T_{j k} \text {. }
$$

The same process is carried out for the second factor, etc. Then relation (27) holds in general because if $i$ and $k$ or $j$ and $k$ are in different classes, the right side of (27) vanishes identically.

From (17) it is seen that $T_{i j}$ is homogeneous of degree -1 so by Euler's theorem

$$
T_{i j}=-\sum_{1}^{n} g_{k} \partial T_{i j} / \partial g_{k}=\sum_{1}^{n} g_{k} T_{i k} T_{j k} .
$$

If all $g_{k}=1$, let $T_{i j}=P_{i j}$. This defines a real symmetric matrix $P$ such that $P^{2}=P$. Thus $P$ is a perpendicular projection matrix of $U$ into a subspace $V$. If we compare (28) and (16) it follows that $\Psi_{i}=T_{i i}$. Thus for all $g_{k}=1: \Psi_{i}$ $=P_{i i}, \Psi_{i j}=-\left(P_{i j}\right)^{2}, \Psi_{i j k}=2 P_{i j} P_{j k} P_{k i}$, etc. Thus all derivatives of $\Psi$ are expressible in terms of $P$.

Let $D=\operatorname{det}\left(G P+P^{\prime}\right), T=P\left(G P+P^{\prime}\right)^{-1}$, and $\psi=\log D$. For $g_{k}=1, G=I$ so that $D=1$ and $T=P$. By Theorem 3 and Theorem 5 it follows that at this point all the derivatives of $\psi$ are expressed in terms of $P$ by the same formulae as for $\Psi$. Thus all derivatives of $\Psi-\psi$ vanish at this point. By Taylor's theorem $\Psi-\psi=$ constant so $D=f\left(g_{1}, \cdots, g_{n}\right) / f(1, \cdots, 1)$.

Theorem 10. A diagonal matrix has the same discriminant in two different subspaces if and only if one subspace is a reflection of the other.

Proof. A reflection of $U$ is defined by a diagonal matrix $R$ with diagonal elements +1 or -1 . Thus $R^{2}=I$. Clearly $P_{r}=R P R$ is the perpendicular projection matrix corresponding to the subspace $V_{r}$, the reflection of $V$. Let $D_{r}$ be the discriminant of $G$ in $V_{r}$. Since $G$ and $R$ are both diagonal, $G R$ $=R G$; thus $D_{r}=\operatorname{det}\left(G R P R+R P^{\prime} R\right)=\operatorname{det} R\left(G P+P^{\prime}\right) R=D$.

Suppose now that two subspaces $V$ and $V^{+}$have the same $D$. Then $-\psi_{i j}=\left(T_{i j}\right)^{2}=\left(T_{i j}^{+}\right)^{2}$ so $T_{i j}^{+}=\delta_{i j} T_{i j} ; \delta_{i j}= \pm 1$. As in the proof of Theorem 9 let $D$ be expressed as a product of irreducible factors. Thus if $g_{i}, g_{j}$, and $g_{h}$ appear in a single factor, $\partial T_{i j} / \partial g_{h}=-T_{i h} T_{j h}$ and the right side does not vanish identically. The same relation holds for $T_{i j}^{+}$so comparison gives $\delta_{i j}=$ 
$\delta_{i h} \delta_{j h}$. Let the variable $g_{h}$ have special significance and define $\delta_{i}=\delta_{i h}$. Hence

$$
T_{i j}^{+}=\delta_{i} T_{i j} \delta_{j}
$$

The same process is carried out for the other factors. Then (29) holds in general because if $i$ and $j$ refer to different factors, $T_{i j} \equiv 0$. When all $g_{i}=1$, then $T$ and $T^{+}$become the perpendicular projection matrices $P$ and $P^{+}$. But (29) is precisely the condition that $P^{+}$be a reflection of $P$.

The following four properties of $T$ are stated without proof: Let $i, j, \cdots, h$ be distinct and let $T^{i j \cdots h}$ be that symmetric minor of $T$ which contains the diagonal elements $T_{i i}, T_{j j}, \cdots, T_{h h}$. Then

$$
\operatorname{det} T^{i j \cdots h}=D_{i j \cdots h} / D \text {. }
$$

Let $S^{i j \cdots h}$ be defined as $T^{i j \cdots h}$ with its diagonal elements removed. Then if the set $i, j, \cdots, h$ has at least two members,

$$
\operatorname{det} S^{i j \cdots h}=\psi_{i j \cdots h} \text {. }
$$

The diagonal elements of $T$ may be regarded as independent variables. Thus

$$
\partial \psi / \partial T_{i i}=-g_{i}
$$

Suppose that $D_{i}$ contains the term $a_{r} g_{r_{1}} g_{r_{2}} \cdots g_{r_{m}}$ and that $D_{j}$ contains the term $b_{r} g_{r_{1}} g_{r_{2}} \cdots g_{r_{m}}$. Then

$$
T_{i j}=D^{-1} \sum_{r} \pm\left(a_{r} b_{r}\right)^{1 / 2} g_{r_{1}} g_{r_{2}} \cdots g_{r_{m}} .
$$

The signs of the terms are chosen so as to satisfy (17).

4. Physical interpretations of the symbolism. First consider an example from static mechanics. An elastic shaft with torsional rigidity constant $g_{i}>0$ is held fixed at one end at the angle $e_{i}$. If the other end is twisted to the angle $v_{i}$, then clearly the elastic potential energy is $(1 / 2) g_{i}\left(v_{i}-e_{i}\right)^{2}$.

Consider a system of $n$ such shafts whose free ends are connected by a chain of gears. This gives rise to linear constraints on the angles $v_{i}$. The gear chain can employ differential gear boxes to perform the operations of addition and subtraction. Thus any constraint of the form $\sum_{1}^{n} c_{i}^{\prime} v_{i}=0$ can be enforced. The magnitudes of the constants $c_{i}^{\prime}$ are determined by suitable gear ratios. Hence $v$ can be made orthogonal to an arbitrary set of vectors. This implies that the constraint subspace $V$ is arbitrary.

A string is wound around each shaft and attached to a weight giving rise to a constant torque $e_{i}^{\prime}$. The total potential energy of the system of shafts and weights is

$$
Q=(1 / 2) \sum_{1}^{n} g_{i}\left(v_{i}-e_{i}\right)^{2}-\sum_{1}^{n} e_{i}^{\prime} v_{i}
$$


The potential energy is a function of the coordinates, so by a general principle of static mechanics equilibrium is attained when $Q$ is stationary. Thus by Theorem 5 the equilibrium position is given by $v=T G e+T e^{\prime}$. Since $g_{i}>0$, it is clear from Theorem 6 that $D \neq 0$. If $v_{i}^{\prime}$ is the torque exerted by the shaft on the constraint mechanism, then the equations of equilibrium are of the form (6). It follows that $v^{\prime} \in V^{\prime}$ and $v^{\prime}=T^{\prime} G^{\prime} e^{\prime}+T^{\prime} e$.

Somewhat less obvious examples occur in dynamic mechanics. Consider a system of $r$ particles subject to a holonomic constraint. The $v_{i}$ are interpreted as the velocity components of the particles relative to a rectangular coordinate system $(x, y, z)$. Thus $n=3 r$. The $g_{i}$ are interpreted as the masses of the particles and we pretend that the mass in the $x, y, z$ directions are different. Thus $E$ is twice the kinetic energy of the particles. It is not necessary that the constraint be linear as only its differential form enters the problem. It turns out that the matrix $T$ gives the effective inverse masses under impulsive forces.

Now consider an electrical interpretation. An electrical network may be described as a set of $n$ two-terminal "branches" arbitrarily interconnected at their terminal points. A point where one or more terminals meet is called a junction. A direction is arbitrarily assigned to each branch. Relative to this direction let $v_{i}$ be the voltage across the $i$ th branch and let $v_{i}^{\prime}$ be the current in amperes flowing through the $i$ th branch. Kirchhoff's first law states that the net current entering a junction point vanishes. Obviously the vectors $v^{\prime}$ which satisfy Kirchhoff's first law form a subspace $V^{\prime}$ of $U$. A current flow of unit strength around a simple closed circuit satisfies the first law. Let the corresponding vector be designated as $w^{\prime}$. Given a $v^{\prime} \neq 0$ it is clear that there is a $w^{\prime}$ and a constant $c$ such that the vector $v^{\prime}-c w^{\prime}$ has fewer nonvanishing components than $v^{\prime}$. Hence a set of $w^{\prime}$ will serve as a basis for $V^{\prime}$.

Kirchhoff's second law states that the sum of the voltages directed around any simple closed circuit vanishes. In vector language $v$ satisfies the second law if and only if $\left(v, w w^{\prime}\right)=0$ for every $w^{\prime}$ [9]. It follows by addition that $\left(v, v^{\prime}\right)=0$. Thus Kirchhoff's laws define orthogonal complementary subspaces $V$ and $V^{\prime}$.

The diagonal discriminant $D\left(g_{1}, \cdots, g_{n}\right)$ of a connected network is given by the following well known rule: The coefficients of the terms of $D$ are all equal. Each term is obtained by forming the product of those $g_{i}$ which form a tree of the network. This rule may be proved by noting that in a suitable coordinate system $\operatorname{det} A$ is a Sylvester unisignant determinant [4]. By Theorem 4

$$
D^{\prime}\left(g_{1}^{-1}, g_{2}^{-1}, \cdots, g_{n}^{-1}\right)=D\left(g_{1}, g_{2}, \cdots, g_{n}\right) / g_{1} g_{2} \cdots g_{n} .
$$

Thus the coefficients of $D^{\prime}$ are all equal and the terms are the products of "tree complements" [6].

These Kirchhoffian subspaces are not arbitrary subspaces because of the 
remarkable property that all of the coefficients of a diagonal discriminant are equal. In another paper the writers will investigate subspaces with this property.

For theoretical reasons it is sometimes desirable to introduce additional non-Kirchhoffian constraints. This may be accomplished by the use of ideal transformers (transformers with cores of infinite permeability). It is then possible to achieve an arbitrary constraint.

Each branch of the network is regarded as having a series voltage generator of voltage $e_{i}$ and a parallel current generator of amperage $e_{i}^{\prime}$. For a direct current network in which the branches obey Ohm's law,

$$
g_{i}\left(v_{i}-e_{i}\right)+\left(v_{i}^{\prime}-e_{i}^{\prime}\right)=0 .
$$

Here $g_{i}>0$ is the conductivity of the branch. For alternating current networks without mutual coupling, (36) is again valid with $g_{i}$ a complex number called the branch admittance. The branch impedance is $g_{i}^{-1}$. The solution of (36) is given by (7) and (8). $T$ may be termed the transpedance matrix and $T^{\prime}$ may be termed the transmittance matrix.

Networks with mutual coupling introduced by transformers may be treated by employing a symmetric $G^{\prime}$ matrix in a well known way [5]. However, a transformer may be given an equivalent circuit consisting of an ideal transformer and two (or three) inductors. In this way it is possible to include networks with transformers under the theory of diagonal $G$ matrices. Of course the constraint is then non-Kirchhoffian. Electron tubes give rise to unsymmetric $G$ matrices.

The relationships brought out by Theorem 6 are of fundamental importance in the synthesis of a network having a prescribed behavior as a function of frequency [1].

\section{REFERENCES}

1. R. Bott and R. J. Duffin, Journal of Applied Physics vol. 20 (1949) p. 816.

2. G. A. Campbell, Transactions of the American Institute of Electrical Engineers vol. 30 (1911) pp. 873-909. Collected papers, 1937, pp. 65-101, pp. 102-111.

3. R. Cohn, Proceedings of the American Mathematical Society vol. 1 (1950) pp. 316-324.

4. R. J. Duffin, Bull. Amer. Math. Soc. vol. 54 (1948) pp. 119-127.

5. — Proceedings of the American Mathematical Society vol. 1 (1950) pp. 233-240.

6. Phillip Franklin, Journal of Mathematics and Physics vol. 4 (1925) pp. 97-102.

7. G. Kron, Tensor analysis of networks, Wiley, 1939.

8. P. Le Corbeiller, Matrix analysis of networks, Harvard University Press, 1950.

9. H. Weyl, Revista Matematica Hispano-Americana vol. 5 (1923) pp. 153-164.

UNIVERSITY OF MiCHIGAN, ANN ARbor, Mich.

Carnegie Institute of Technology, Pittsburgh, Pa. 